\title{
EL CONSUMO PROMEDIOY LA ROTACIÓN COMO EFECTOS GENERADORES DE LAS VENTAS EN RESTAURANTES DE LA CIUDAD DE QUITO, ECUADOR
}

\author{
PABLO DÍAZ DUQUE \\ Universidad Tecnológica Equinoccial, Ecuador \\ pvdiaz@ute.edu.ec \\ CAMILA ALEJANDRA BURBANO ARGOTI \\ Universidad Tecnológica Equinoccial, Ecuador \\ camila.burbano@ute.edu.ec \\ DIEGO SALAZAR DUQUE \\ Universidad Tecnológica Equinoccial, Ecuador \\ diegoa.salazar@ute.edu.ec
}

\section{RESUMEN}

Uno de los principales procesos en un restaurante es aquel relacionado con la gestión de venta desde que se ofrece un producto hasta que un cliente lo consume. Para esto, existen elementos que el cliente no percibe y que intervienen en la decisión de repetir la compra; es necesario que el administrador conozca qué elementos influyen en este proceso. Para este estudio se ha planteado establecer si existe relación entre el consumo promedio y la rotación como elementos orientadores de la preferencia de un cliente y del nivel de ventas del establecimiento. Esto se logra identificando los factores que influyen en el cliente durante la toma de decisiones y contribuye a establecer estrategias para elevar el nivel de ingresos, considerando a la rotación y al consumo promedio como variables diferenciadoras de la gestión de establecimientos.

PALABRAS CLAVE: RESTAURANTES, CONSUMO PROMEDIO, ROTACIÓN, VENTAS.

\section{ABSTRACT}

One of the main processes in a restaurant is that related to sales management, since a product is offered until a customer consumes it, for this there are elements that the client does not perceive them and that intervene in the decision to repeat the purchase; for this it is necessary to know, on the part of the administrator, which elements influence in this process. The purpose of this study was to establish if there is a relationship between average consumption and turnover as guiding elements of a customer's preference and the level of sales of the establishment by identifying the factors that influence the customer during decision making; this contributes to set strategies to raise the income level, considering the rotation and the average consumption as differentiating variables of the establishment management.

KEYWORDS: RESTAURANTS, AVERAGE CONSUMPTION, ROTATION, SALES. 


\section{INTRODUCCIÓN}

Los establecimientos de alimentos y bebidas son importantes generadores de ingresos para la industria del turismo. Contribuyen de manera directa con la cadena de valor de los alimentos, desde que se adquieren como materia prima, se procesan y se sirven a los clientes (en este caso, son valorados como comensales). Este proceso, necesario para la operación del negocio, genera obligaciones monetarias que deben ser cubiertas por el establecimiento a través de los ingresos obtenidos por la gestión de ventas y que, al igual que en cualquier otro negocio, permiten cubrir los gastos de operación y de administración de la empresa y en el caso de que las ventas superen los costos, se puede generar un excedente que se constituye en la utilidad del negocio. Por otro lado, los clientes de restaurantes pagan por su experiencia gastronómica, la cual engloba todo el concepto que ofrece el establecimiento; si esta experiencia es positiva, se genera lealtad y la posibilidad cierta de que se repita la compra, lo que permite al establecimiento contar con los recursos necesarios para cumplir sus obligaciones.

El objetivo de la investigación fue estudiar la relación entre el consumo promedio y la rotación como variables generadoras de las ventas en restaurantes de la ciudad de Quito, en los sectores Centro Histórico, República del Salvador, La Mariscal e Iñaquito, lo cual permitirá establecer la importancia y el impacto de estas variables en la generación de los ingresos en estos establecimientos. Es decir, el administrador del negocio deberá planificar estrategias comerciales y de servicio orientadas a alcanzar un mercado objetivo y lograr posicionamiento de marca en la mente de los clientes. Lo anterior con la finalidad de incrementar el número de visitas, el nivel de consumo promedio en el local y, por supuesto, lograr que el consumidor salga satisfecho y dispuesto a vivir esa experiencia en otra oportunidad cercana.
Existen diversas posturas que abordan este tipo de temática pero que no alcanzan a contribuir de forma efectiva con el objetivo de esta investigación. Aun así, se consideran como referentes los aportes de Bustos y González (2006); Zamora, Vásquez, Díaz y Grandón (2011); Cabrera (2013); Moliner y Berenguer (2011); y la UIEM, Puebla y Maldonado (2014). El presente estudio aborda, en primer lugar, los conceptos y fundamentos referentes a las ventas, consumo promedio y rotación en establecimientos de alimentos y bebidas y, en segundo lugar, se explica la metodología que se manejó como parte del diseño de investigación empírica utilizada para la comprobación de la hipótesis para, finalmente, presentar los resultados alcanzados.

\section{REVISIÓN DE LA LITERATURA E HIPÓTESIS}

\section{LOS RESTAURANTES EN LA CIUDAD DE QUITO}

El sector de alimentos y bebidas es una actividad de servicio que forma parte del sistema turístico y que deja un fuerte ingreso económico según el lugar geográfico donde se desarrolle (Boullón, 2003; World Tourism Organization (UNWTO), 2015); comprende, por lo general, empresas que ofrecen productos y servicios según las preferencias y demandas gastronómicas del sector de alimentos y bebidas del mercado. Dentro de esta actividad hay establecimientos sub (actividades secundarias) con otras actividades como restaurantes, bares, fuentes de soda y cafeterías, que se clasifican en: de lujo, primera, segunda, tercera y cuarta categoría (CAPTUR, 2002).

De acuerdo con el Consolidado Nacional o Catastro del Ministerio de Turismo del Ecuador, en el año 2013 se registraron 12967 establecimientos de alimentos y bebidas en el país. De ellos, la gran mayoría son restaurantes y 1920 corresponden a la ciudad de Quito. Para el año 2014, el sector gastronómico tuvo un incremento anual 
del 9\% lo que da un total de 2093 restaurantes en la ciudad. De estos, la mayor concentración de restantes se encuentra ubicada en los sectores del Centro Histórico, República del Salvador, La Mariscal e Iñaquito.

Estos establecimientos ofrecen productos variados y diversos con una gastronomía nacional o internacional muy amplia. Tienen una infraestructura adecuada, personal preparado y conceptos gastronómicos innovadores que les permiten generar ingresos y diferenciarse competitivamente de otros establecimientos similares.

Hay que considerar que los ingresos en los restaurantes pueden ser muy variados y dispersos, dependiendo de los días de la semana o las distintas temporadas que existen en el año. Por lo tanto, para que se generen ingresos sostenidos, las empresas deberán tomar en cuenta aspectos como: ubicación geográfica, oferta gastronómica, nivel de precios, calidad de producto y servicio, valor y beneficios identificados para satisfacer las necesidades y expectativas del cliente, así como las diversas estrategias comerciales aplicadas, las diferentes motivaciones de compra, pero sobre todo la satisfacción experimentada por un consumidor cuando visita el establecimiento.

\section{EL PROCESO DE VENTAS}

Durante mucho tiempo, las ventas han jugado un papel importante en la economía del mercado, no solo porque generan ingresos para las empresas, sino porque también permiten medir el comportamiento del consumidor sobre sus necesidades, gustos y preferencias al momento de una compra. La literatura manifiesta que las ventas se basan en una serie de técnicas de comunicación y conocimientos prácticos para informar de los beneficios que pueden generar diversos productos, servicios y el intercambio a favor de ambas partes (consumidor-empresa) (De la Parra, 2004). Por lo tanto, el concepto de ventas puede ser visto desde dos enfoques; el primero, desde la perspectiva empresarial, la cual determina estrategias comerciales que promueven la compra de un producto o servicio por medio del intercambio entre un bien o servicio por un valor monetario pactado. El segundo, desde la perspectiva del consumidor como un proceso por el cual un cliente consigue un objeto o servicio deseado que satisfaga sus necesidades (Ninamango Guevara, 2012).

Para que se genere este intercambio, tanto las empresas como los clientes deben desarrollar un acuerdo mutuo de convivencia en donde se beneficien las dos partes. Es decir, la empresa, por medio de la gestión comercial (Baskerville \& Dulipovici, 2006), debe cubrir expectativas del cliente y generar ingresos. Por su parte, el cliente debe realizar un proceso de transacción económica de forma continua por medio de la compra que le retribuyan beneficios y valores (Kahle \& Kennedy, 1989; Kotler, 2012). De acuerdo con León Valbuena (2013), se debe proporcionar a los compradores un producto y un servicio por el que vale la pena pagar con el mejor valor representado en la combinación atractiva de precio, características y calidad de servicios.

En efecto, las empresas deberán tomar en consideración factores como el consumo del cliente y el número de veces que un consumidor adquiere un producto o servicio. En el sector de alimentos y bebidas, las ventas deben ser consideradas como un proceso de gestión importante, ya que de ellas se generan ingresos y a su vez rentabilidad. Aquellas empresas que tomen este enfoque de forma superficial o a la ligera, se verán afectados a corto o mediano plazo en los resultados financieros; por lo tanto, directivos o responsables de empresas dedicadas a la restauración (restaurantes) deben identificar y controlar factores de éxito que generen más contribución de dinero para la empresa (Davis B. L., 2013). Es decir, se debe considerar cuánto es el consumo que realiza un cliente en el estable- 
cimiento y cuántas veces se genera ese consumo para medir las ventas.

En síntesis, se conoce que las ventas tienen relación directa con los ingresos (León Valbuena, 2013) por lo tanto, están influenciadas por factores que pueden ser controlados por la empresa. Entre los factores que influyen para la generación de ventas se tienen la rotación y el consumo promedio. Partiendo de que existe una relación entre el consumo promedio y la rotación para la generación de las ventas, se establece la siguiente hipótesis:

H1: Existe una relación entre los factores que influyen en el consumo promedio y los factores que influyen en la rotación para la decisión de compra en restaurantes de la ciudad de Quito.

\section{EL CONSUMO PROMEDIO}

Uno de los principales aspectos que se debe considerar dentro de un sistema complejo de costos, y que se puede pasar por alto en la mayoría de situaciones, es el cálculo del consumo promedio (CP). Cabe recalcar que, para abordar esta temática no existen estudios que profundicen su aplicación en forma integral, lo que conlleva a desarrollar su análisis conceptual de manera independiente: consumo y promedio y que se desarrollan con mucha frecuencia en el sector hotelero o de alimentos y bebidas (Davis B. L., 2013). Posiblemente, su uso empírico ha sido manejado a lo largo del tiempo, siendo muy difícil identificar cuándo se aplicó por primera vez. Lo que sí se puede establecer es que, a través de su resultado, se pueden generar estrategias que mejoren los indicadores proyectados por la empresa o negocio para su beneficio. Con este antecedente, conviene realizar un análisis de estos términos.

En primer lugar, se tiene el término de consumo; según la Real Academia Española (2012), para este tipo de estudio se lo denomina como el acto de "utilizar comestibles u otros bienes para satisfacer necesidades o deseos". Este proceso depende de la disponibilidad y variedad de alimentos que existen en un determinado sector geográfico o lugar y que influye en la seguridad alimentaria y bienestar nutricional (Pedraza, 2005; Segura \& Varó, 2010; Mataix Verdú, 2013). Para esta investigación, se hace referencia al consumo de alimentos o bebidas dentro de un restaurante. Es decir, lo que para algunos es un acto fisiológico continuo de alimentarse, para otros como Feijoó et al. (2009), es el flujo de producción de alimentos el cual se inicia con la compra de materia prima y que luego de ser objeto de un minucioso sistema de procesos finaliza con el cobro de un servicio prestado.

Por otro lado, se tiene el término promedio, o promedio ponderado. Este es una forma de localizar la parte central de un conjunto (Barrantes, 2003). El resumen de los datos que se obtienen de este a nivel estadístico puede realizarse en forma tabular, gráfica o numérica (Rodríguez Franco \& Pierdant Rodríguez, 2014). El cálculo más común que se maneja para este procedimiento es la sumatoria de cada uno de los valores que son parte de los componentes de un conjunto, dividido para el número de componentes que existe en dicho conjunto (Rodríguez Franco \& Pierdant Rodríguez, 2014).

A nivel de restaurantes, el promedio es un indicador que se concentra en un cálculo a partir del número de cubiertos servidos, ingresos y costos promedios de platos y costos promedios de servicios por cliente (Ferrer \& Gamboa C., 2005). Ferrer y Gamboa (2005) resaltan que el promedio también es un indicador que se calcula a partir del ingreso promedio por cliente y el nivel de ocupación por restaurante. De manejar este procedimiento a nivel de alojamiento, se destinará a partir del servicio por habitación. Feijoó et al. (2009) determinan que el consumo promedio surge de la relación que existe entre el costo-volumen-utilidad y que está relacionado con la variable volumen. 
Para el cálculo de este procedimiento se deberá identificar qué tipo de consumo promedio se desea obtener, considerando si el restaurante se encuentra en actividad o en proyecto. El primer resultado de consumo promedio es el que se obtiene al momento en que un cliente realiza una transacción; a partir de la sumatoria del precio de cada uno de los platos que se consumen, en un solo servicio, por cliente y a partir de este, el promedio entre todos los clientes en un determinado tiempo (Cuevas, 2002; Barrantes, 2003). El segundo resultado se obtiene a partir de la sumatoria de cada uno de los precios fijados para los platos que conforman la carta del restaurante dividido por el número de platos existentes (Feijoó \& et al., 2009). Por último, a partir del histórico de ventas producidas en un determinado tiempo dividido por el número de clientes que acudieron al establecimiento en este tiempo (Feijoó \& et al., 2009). La tabla 1 muestra, de una forma más didáctica, cada tipo de cálculo.

\section{Criterios que influyen para el consumo promedio (CP)}

Una de los principales variables que influye en las ventas en un restaurante es el consumo promedio. Este indicador tiene estrecha relación con varios criterios que, al ser considerados o no por el administrador, afectarán al resultado final de la operación del negocio y cuyo impacto deberá ser evaluado a corto, mediano o largo plazo. Entre los criterios que se presentan como factores que intervienen en el consumo promedio están:

a. Infraestructura del establecimiento. Gomes, De Azevedo Barbosa y Gomes de Souza (2013) citan a Heung y Tianming (2012) y Lemoine (2003) y resaltan que existe un aspecto importante que puede afectar o favorecer a los empresarios. Se trata de la creación de una infraestructura con espacios físicos acordes a la oferta en el punto de venta. La estructuración de un diseño acogedor y funcional es una alternativa cada vez más usada por las empresas de servicios e influye al tipo de producto y servicio que se está ofreciendo e identificando el valor por el cual un cliente está dispuesto a pagar.

En los establecimientos de alimentos y bebidas se deben manejar conceptos que acompañen a la infraestructura y al ambiente del restaurante. Este diseño debe ser estructurado correctamente, ya que en muchos de los casos la distribución de las mesas, la cocina, las estaciones de servicio, entre otros, puede generar un servicio más rápido o en su defecto, lo contrario (Cooper, Floody, \& Mc Neill, 2002). Para otros autores, como Jorge Vera y Andrea Trillo (2009), la calidad en el servicio es medida por medio

TABLA 1

CÁLCULO DEL CONSUMO PROMEDIO PARA RESTAURANTES EN ACTIVIDAD

\begin{tabular}{|l|l|}
\multicolumn{1}{|c|}{ Encargado } & Potestades \\
\hline Consumo Promedio por cliente & $\mathrm{CP}=\frac{1}{n} \sum_{i=1}^{n} a i=\frac{(a 1+a 2+a 3+\cdots a n)}{n}$ \\
\hline Consumo Promedio según carta & $\mathrm{CP}=\frac{1}{n} \sum_{i=1}^{n} a i=\frac{(a 1+a 2+a 3+\cdots a n)}{n}$ \\
\hline Consumo promedio según el total de clientes & $\mathrm{CP}=\frac{\sum V T}{\sum c T}=\frac{V T d 1+V T d 2+V T d 3+\cdots+V T d n}{c T d 1+c T d 2+c T d 3+\cdots+c T d n}$ \\
\hline
\end{tabular}

Nota: $(\mathrm{P}=$ consumo promedio; $\mathrm{a}=$ precio del producto; $\mathrm{n}=$ número de sumandos; $\mathrm{VT}=$ =ventas totales; $\mathrm{d}=\mathrm{d}$ ías; $\mathrm{C}=$ =clientes totales.

Fuente: Elaboración propia. 
de varias dimensiones y variables como: las instalaciones (compuestas por las características físicas del establecimiento), la comodidad que brindan las instalaciones, la accesibilidad de parqueaderos, la higiene y la ubicación.

b. Servicio de calidad. Según Cooper, Floody y Mc Neill (2002) y Cabrera (2013), un cliente frecuente representa una excelente oportunidad publicitaria para el restaurante. Es importante conocerlos para identificar de mejor manera sus necesidades y entregar siempre la calidad y el servicio que están esperando. Aquí cabe hacer la pregunta ¿existe asesoramiento sobre el producto por parte del encargado de la venta en un restaurante (mesero)? Posiblemente, la respuesta por parte de los administradores es afirmativa y es una inquietud que tienen todos los encargados de gestionar un establecimiento de forma constante; mientras algunos conocen la respuesta, otros creen conocerla y consideran que su personal desarrolla sus actividades con calidad, lo podría no ser así.

El proceso de evaluar si existe o no el asesoramiento adecuado es muy complejo. Medir la actitud del mesero constantemente llega a ser un hostigamiento que, a la larga, no favorecerá a la calidad del servicio. Por lo tanto, este aspecto deberá ser medido al momento de identificar y contratar en un futuro personal que desarrollará las actividades de servicio y así detectar si la nueva fuerza laboral posee o no un espíritu de servicio (Suárez \& et al., 2011).

Uno de los procesos clave que se aconseja para un servicio de calidad es la personalización. S trata de un sistema que ya se gestiona y se oferta de forma eficiente en restaurantes de alta gastronomía y por el que la gente está dispuesta a pagar un alto valor por reci- bir lo que espera. Para Gomes, De Azevedo Barbosa y Gomes de Souza (2013), la personalización es un atributo clave del servicio. Un cliente que mide su experiencia de servicio recibido ayuda a superar el estándar que se brinda en el establecimiento; esto motiva al personal a fortalecer los procesos, superar las expectativas de los clientes y cumplir objetivos de la empresa por medio del empowerment. La percepción del servicio por parte de los clientes es medida por dos variables: las expectativas de los clientes y los estándares de calidad (Kandampully, Mok, \& Sparks, 2009).

c. Oferta gastronómica en forma integral. Un fenómeno que se produce en el sector de alimentos y bebidas es la constante evolución que se suscita sobre los gustos, preferencias y necesidades que se producen en el cliente, sobre todo por los cambios que se generan a nivel mundial y las nuevas tendencias que surgen paulatinamente, lo que obliga a los establecimientos (restaurantes) a adaptar, mejorar y trasformar sus productos y servicios con la finalidad de cubrir las demandas del mercado. El producto gastronómico que se presenta en los establecimientos de alimentos y bebidas es muy amplio, tanto es así que, dependiendo del sector geográfico donde se ofrezca y el tipo o categoría del establecimiento, se pueden encontrar varios platillos culinarios preparados, más todas aquellas bebidas alcohólicas y no alcohólicas que se puedan imaginar: aperitivos, vinos, cervezas, espirituosos y licores, cubriendo necesidades y preferencias de los consumidores. Una de las herramientas que facilita la decisión del cliente es la carta, que desarrollada y estructura adecuadamente estimula al cliente a elegir uno o varios platillos ya sean estos una entrada, un postre o una bebida, mismos que pueden acompañar al plato fuerte. 


\section{LA ROTACIÓN DE CLIENTES (R)}

Otro de los principales cálculos que se deben tomar en consideración dentro de un restaurante es la rotación de clientes. Este es un proceso que se genera por el efecto de la circulación de clientes que frecuentan el establecimiento en un determinado tiempo y que se mide a partir de la ocupación que se genera en cada mesa o de cada puesto (Martínez, 2004), representado como uno de los indicadores que se relacionan con los estados financieros. A mayor rotación de clientes resulta más beneficioso para el restaurante, pues genera mayores ingresos. Este factor se obtiene a partir de la información (Choo, 2002) estadística que se visualiza a partir de las comandas, logrando identificar cuántas veces se atendió la misma mesa o cuantas veces se sentó una persona en una silla, ya sea en función del turno, las horas de mayor trabajo o movimiento, el servicio o el día.

Es decir, para calcular, es suficiente con dividir el número de clientes registrados en una unidad de tiempo para la capacidad máxima que posee el establecimiento. Este efecto se puede desarrollar a partir de la ocupación que se genera en el horario de desayuno, almuerzo o cena (Martínez, 2004). El resultado que este cálculo arroje medirá la productividad con la que opera la empresa (Campos, 2010). El valor óptimo que debería alcanzar este efecto deberá superar el número de puestos con los que cuenta el establecimiento. Lo que más adelante será superior en 1 o su equivalente al 100\%.

Por otra parte, la rotación de clientes propicia la oportunidad de identificar la satisfacción de comensales (Fernández, 2010), esto permite planificar a futuro estrategias oportunas (Rodés, 2014) que generen productos y servicios que desarrollen una lealtad a largo plazo (Cabrera, 2013, p. 155). La tabla 2 muestra, de una forma más didáctica, el cálculo de la rotación.

\section{TABLA 2 \\ CÁLCULO DE LA ROTACIÓN DE CLIENTES EN RESTAURAN- TES CON ACTIVIDAD POR DÍA}

\begin{tabular}{|c|c|}
\hline Rotación & Potestades \\
\hline \multirow{3}{*}{ Rotación de clientes } & $\mathrm{R}=\frac{\sum c}{\sum p}=\frac{c 1+c 2+c 3+\cdots c n}{p 1+p 2+p 3+\cdots p n}$ \\
\hline & $\mathrm{R}=\frac{\text { total de clientes }}{\text { total de puestos }}$ \\
\hline & $R=n$ veces \\
\hline
\end{tabular}

Nota: $c=$ clientes; $p=$ puestos; $n=$ número de sumandos; $S i$ Res $>1$, el resultado es óptimo sobre el total de puestos.

Fuente: Elaboración propia.

El resultado obtenido indica la ocupación del establecimiento en un periodo determinado. Si este valor supera la base de 1, se considera que estuvo lleno de clientes en dicho horario, lo que determina una buena rotación y excelente operatividad. Aunque siempre sería bueno superar esa base de 1 y tener clientes que esperen para ser atendidos, lo que obligaría a establecer estrategias para tener siempre el local lleno y, al mismo tiempo, brindar productos y servicio de excelente calidad.

\section{Criterios que influyen en la rotación}

Así como el consumo promedio es un indicador que interviene en el resultado de las ventas, la rotación también se suma como un efecto que influye en sus resultados. Para entender qué criterios son los que afectan a la rotación es necesario hacerse una pregunta: ¿Qué hace que un cliente regrese a un restaurante? Para responder a esta incógnita, es necesario identificar los aspectos que intervienen en la rotación, donde la lealtad tiene un peso muy importante y donde los atributos que más valoran, como el precio, tiempo de atención, localización, diseño y calidad de la comida como producto son un factor muy importante (Schmal \& Olave, 2014).

a. Diseño y elementos sensoriales. De acuerdo con Gomes, De Azevedo Barbosa y Gomes 
de Souza (2013), uno de los criterios que el empresario o administrador deberá tomar en consideración para que exista una adecuada rotación son los distintos elementos sensoriales. Estos son adaptados para la satisfacción y comodidad tanto de los clientes como del personal interno; tanto así, que pueden influir en la calidad del producto y el servicio. La percepción visual, olfativa, auditiva, gustativa, táctil o de temperatura tienen una estrecha relación con el diseño o el ambiente que posee un negocio de alimentos y bebidas (Cabrera, 2013), los cuales se revelan "como instrumentos capaces de recrear un universo de consumo hedonista y responder a las motivaciones afectivas de los individuos" (p. 338) y generar experiencias únicas en el cliente.

Gomes, De Azevedo Barbosa y Gomes de Souza (2013) esclarecen que no todos los restaurantes se dedican solo a preparar alimentos culinarios que gusten y satisfagan las necesidades de los clientes, sino que crean espacios únicos que permiten sentir cómodos a quienes los vistan y se benefician de sus servicios. En ellos, uno de los personajes o empleados más representativos es el chef, quien interactúa con los productos gastronómicos como creador de los elemento clave al momento de preparar platillos y que influye a nivel sensorial en el cliente o comensal.

Respecto a este tema, Rieunier (2004) y Bellos y Kavadias (2011) mencionaron que el administrador del restaurante puede ofrecer a sus clientes la oportunidad de sentir la calidad del ambiente a través de sus sentidos e influenciar positivamente en su decisión de visitar nuevamente el establecimiento y generar fidelidad a largo plazo. Como consecuencia, el sabor, la textura, los olores, entre otros factores, a través de las percepciones sensoriales, son aspectos claves que influyen en el cliente para la decisión de compra. Como ejemplos de ello se pueden resaltar: un producto gastronómico, el cual se encuentra armonizado por los sentidos al ser probado y que puede ser guardado en la memoria del cliente como un recuerdo (Ocampo, 2012) o durante el proceso de decisión de compra, donde muchas veces el lugar, el diseño o la atmósfera del negocio genera una influencia más grande que el mismo producto (Milliman, 1986). Un claro ejemplo de ello son los negocios en el entorno que percibe un cliente que acude a un establecimiento de alimentos y bebidas en la ciudad de Quito, sobre todo en su Centro Histórico, el cual posee ciertas peculiaridades arquitectónicas que complementan a la gastronomía, resaltando sus esencias culinarias y conectado con la percepción del consumidor.

b. El tiempo de espera y atención en el servicio. Uno de los aspectos que afectan a la rotación es el tiempo que se produce desde que el cliente entra al restaurante hasta que se sirve un plato y paga. Dependiendo del tipo de servicio, el tiempo de espera puede ser percibido por el cliente de una manera positiva o negativa; por ejemplo, en restaurantes de comida rápida, el tiempo corto en el servicio es vital, mientras que, en restaurantes de lujo, se manejan tiempos amplios debido a la variedad de alimentos y bebidas que se degustan en estos servicios (Cabrera, 2013). Es decir, las distintas actividades y pasos que se originen al momento de ingresar, tomar la orden y entregar el pedido pueden o no generar un valor agregado; si se procede inadecuadamente, puede caer en el desarrollo de tiempos muertos.

Una mala experiencia genera una barrera o un muro entre el cliente y el establecimiento, lo que influye a largo plazo en la lealtad (Cabrera, 2013) y, por ende, una rotación que 
tiende a la baja. Por otro lado, para Zamora, Vásquez, Díaz y Grandón (2011), la satisfacción de un cliente genera lealtad, alta posibilidad de recompra y la certeza de que sus comentarios siempre van a ser escuchados y orientados siempre a mejorar el servicio que ofrece el establecimiento. Gomes, De Azevedo Barbosa y Gomes de Souza (2013) aseguran que para los establecimientos que ofertan servicios basados en los sistemas de personalización, ofrecer los productos durante el servicio es el momento donde se genera la experiencia y más si el tiempo empleado en la atención es el adecuado, tanto para la experiencia del cliente como para el desarrollo de actividades del personal. Por lo tanto, un alto nivel de demanda representado por una alta rotación debe ser enfrentada eficientemente con una disminución de los tiempos de servicio, tal como lo señalaron Schmal y Olave (2014).

c. Ubicación e Infraestructura. Uno de los criterios que un cliente evalúa para volver a un restaurante es la ubicación geográfica; es decir, si se encuentra en un lugar cercano, seguro, con buenas vías de acceso (Kotler, 2012) y que posea parqueadero ocasionará que el cliente regrese. En el caso contario, buscará a futuro otro lugar en el cual se sienta más cómodo. Esto afecta considerablemente a la rotación del establecimiento y a la lealtad. Por otro lado, existen clientes que, a pesar de estos limitantes, evalúan otros indicadores con mayor peso como el sabor de la comida y la atención, lo que genera lealtad y aporta a la rotación.

d. Producto y servicio con calidad y cantidad. Según Sandra Cabrera (2013), para que exista una lealtad, los clientes comparan los productos y el servicio con experiencias obtenidas de otros restaurantes; sobre todo si se trata de una calidad mejor y de percibir una porción con mayor volumen a un precio justo y razonable. Un buen producto y un buen servicio constituyen la fórmula perfecta para el éxito.

e. El precio. Posiblemente uno de los criterios que un cliente evalúa y que no necesariamente es el más importante, es el precio. Por ejemplo, la experiencia profesional ha demostrado que la comida rápida posee un precio muy económico para un segmento de mercado variado, por lo que genera una mayor rotación, sobre todo al ser un servicio que se consume en un tiempo muy corto. Por otro lado, aquellos establecimientos que ofertan alta cocina brindan productos con precios elevados a un mercado más segmentado (Kotler, 2012) y en un lapso de tiempo más amplio y, por lo tanto, al ser un servicio personalizado el precio es más alto. El precio deberá estar acorde con el tipo de establecimiento, producto y servicio que se oferte. Sin embrago, una investigación de Njite, Dunn y Kim (2008) concluyó que el precio de venta en restaurantes de lujo es una variable de menor importancia frente a la relación de los clientes con el establecimiento.

f. Las Promociones. Otro de los criterios que evalúa el cliente y que influye en la rotación son las promociones; ya sea para informar sobre las distintas actividades que desarrollan o las diversas ofertas que poseen, así como lograr persuadir al mercado para el desarrollo de la compra (Kotler, 2012).

g. La Innovación. Cabrera (2013) sostiene que un negocio se debe innovar permanentemente; de no cambiar, puede promover al fracaso de muchos restaurantes. La innovación satisface las necesidades de la gente, por lo tanto, el desarrollo de nuevas ofertas recae en el retorno del cliente. Frente a esto, Cabrera (2013) señala que la innovación se puede poner de manifiesto en los 
diferentes platos de la carta, en detalles de su concepto o en los diferentes servicios adicionales que pueda ofrecer el establecimiento a sus clientes. De la misma manera, la innovación puede estar enfocada en función de las herramientas tecnológicas que los establecimientos utilicen con sus clientes. A partir de una investigación realizada por la Universidad de Cornell se pudo determinar que la herramienta tecnológica más utilizada en los Estados Unidos durante el año 2008 fue la reservación en línea, mientras que el pago mediante los teléfonos celulares fue la menos usada (Dixon, Kimes, \& Verma, 2009).

\section{METODOLOGÍA}

El desarrollo de esta investigación es de naturaleza descriptiva exploratoria correlacional, con un diseño multivariado. Se enfoca en elementos que tienen una estrecha relación con el consumo promedio y la rotación como factores que influyen en la generación de las ventas en restaurantes de alimentos y bebidas de la ciudad de Quito en los sectores: Centro Histórico, República del Salvador, La Mariscal e Iñaquito. Estos lugares fueron establecidos para la captura de los datos primarios a través de entrevistas personales en los establecimientos gastronómicos de cada sector.

Para ello, se diseñó un cuestionario que se estructuró en dos partes. La primera incluye los aspectos generales del cliente, que permiten avalar la información básica para desarrollar la encuesta (sexo, edad, formación académica, ocupación). La segunda parte está desarrollada en dos tablas a partir de las variables consumo promedio y rotación. La primera variable fue medida a través de tres criterios: la infraestructura del establecimiento, el servicio de calidad y la oferta gastronómica, relacionados con el precio que el cliente está dispuesto a pagar. La segunda variable medida a partir de diez criterios: el diseño, el tiempo de espera, la atención en el servicio, la ubicación geográfica, la infraestructura interna y externa, la calidad del producto, la cantidad de producto, el precio, las promociones y la innovación. En todos los criterios se utilizaron escalas con preguntas tipo Likert de cinco posiciones, para evaluar el grado de acuerdo o desacuerdo con las proposiciones establecidas.

\section{Análisis de fiabilidad}

Como parte del proceso de investigación, se validó primero un cuestionario a tres especialistas del sector gastronómico, los cuales debían cumplir criterios como: nivel de formación universitaria, desempeño laboral en el campo de la docencia universitaria o del sector de alimentos y bebidas y que sean investigadores con experiencia en la temática presentada. De esta prueba se realizó un ajuste en una pregunta, pues inicialmente se solicitaba analizar el producto y el servicio como un solo criterio, cuando es necesario estudiar a cada uno por separado.

Una vez validado el cuestionario, se aplicó el instrumento en la muestra. Para determinar la confiabilidad, se aplicó el índice alfa de Cronbach. Para ello, se utilizó el programa estadístico Statistical Package for the Social Sciences (SPSS), que comprobó que no existen diferencias importantes para cada ítem (elemento de medición). Ello confirma lo validado en la prueba piloto. Los valores de la fiabilidad de las escalas Alfa de Cronbach y la correlación elemento total corregida se presentan en la tabla 3. La fiabilidad de las escalas para las ventas excede en sus dos enfoques a .80, el mínimo recomendado por Oviedo y Campo (2005) para investigaciones.

El estudio se realizó a las personas residentes de Quito, en un segmento de mercado comprendido entre 1062476 personas (502 179 hombres y 
TABLA 3

ANÁLISIS DE FIABILIDAD DE VENTAS

\begin{tabular}{|l|l|l|}
\hline \multicolumn{1}{|c|}{ Vescripciones de la escala de } \\
\multicolumn{1}{|c|}{ Ítem } & $\begin{array}{l}\text { Alfa de } \\
\text { Cronbach }\end{array}$ & $\begin{array}{l}\text { Correlación } \\
\text { elemento } \\
\text { total } \\
\text { corregida }\end{array}$ \\
\hline Ventas (V) & $\mathbf{0 . 8 4 4}$ & \\
\hline Consumo Promedio (CP) & $\mathbf{0 . 8 1 9}$ & \\
\hline Infraestructura versus precio & & 0.668 \\
\hline Calidad del producto versus precio & & 0.709 \\
\hline Oferta gastronómica versus precio & & 0.647 \\
\hline Rotación (R) & $\mathbf{0 . 8 3 6}$ & \\
\hline Diseño & & 0.609 \\
\hline Tiempo de espera & & 0.526 \\
\hline Atención en el servicio & & 0.560 \\
\hline Ubicación geográfica & & 0.541 \\
\hline Infraestructura interna y externa & & 0.577 \\
\hline Calidad del producto & & 0.530 \\
\hline Cantidad de producto a ofrecer & & \\
\hline Precio de un producto y servicio & & \\
\hline Promociones & & \\
\hline Constantes innovaciones & & \\
\hline
\end{tabular}

Fuente: Elaboración propia.

560297 mujeres, según la Secretaría de Territorio, Hábitat y Vivienda - MDMQ, 2010) con edades entre 18 años a más que suelen frecuentar restaurantes de los sectores: Centro Histórico, República del Salvador, La Mariscal e Iñaquito. Se toma en consideración este universo de carácter infinito ya que el consumidor quiteño no frecuenta ir solo a un restaurante cercano a donde el habita, por lo que prefiere trasladarse a otros sectores de la ciudad según ya sea por motivo de trabajo, estudios, gustos, accesibilidad, disponibilidad o recursos.
A partir de esta base, se determinó un tamaño de muestra de 385 personas aplicando un margen de error de muestra del 5\%, un nivel de confianza del 95\% ( $z=1.96)$ y una heterogeneidad del 50\%. Este proceso se realizó entre semana y los fines de semana por considerar que existen diversos segmentos de mercados que frecuentan estas zonas según el día. De este proceso, solo el $\mathbf{9 6 . 3 1 \%}$ de encuestas fueron validadas (370 encuestas) debido a inconsistencias y respuesta duplicadas. En la tabla 4 se muestra la ficha técnica de estudio.

\section{RESULTADOS}

Para analizar los resultados generales, según el trabajo de campo, es necesario primero, presentar los datos obtenidos según el método aplicado de la encuesta personal. De la muestra identificada se alcanzó a realizar un estudio a 221 personas de sexo masculino y 149 de sexo femenino (370 personas). El número de encuestados

\section{TABLA 4 \\ FICHA TÉCNICA DEL ESTUDIO}

\begin{tabular}{|l|l|}
\hline Población & $\begin{array}{l}\text { 1062 } 476 \text { personas (grupos de edades } \\
\text { mayores a 18 años) }\end{array}$ \\
\hline Ámbito & $\begin{array}{l}\text { Centro Histórico, República del } \\
\text { Salvador, La Mariscal e lñaquito, Quito } \\
\text { - Ecuador }\end{array}$ \\
\hline Método para levantar datos & Encuesta personal \\
\hline Respuesta a encuestas & $\begin{array}{l}\text { Clientes que frecuentan y consumen } \\
\text { alimentos y bebidas }\end{array}$ \\
\hline Tamaño de la muestra & 384 encuestados (100\%) \\
\hline Tasa de respuesta & $96.31 \%$ encuestas fueron válidas \\
\hline Margen de error de muestra & $5 \%$ \\
\hline Nivel de confianza & $95 \%$ (z=1.96) \\
\hline Heterogeneidad & $50 \%$ \\
\hline Trabajo de campo & 1 al 15 de febrero de 2017 \\
\hline
\end{tabular}

Fuente: Elaboración propia. 
según los rangos de edades fueron: 81 personas hasta 20 años, 102 que corresponden a edades entre 20 a 25 años, 74 entre 26 a 30 años, 41 entre 31 a 35 años, 26 entre 35 a 40 años, 24 de 41 a 45 años y 22 con más de 45 años.

En concordancia con lo expuesto en el apartado anterior, los resultados analizados están basados en criterios que tratan de estudiar, si existe relación entre el consumo promedio y la rotación. Para ello, se analizó primero la media y desviación estándar de cada uno. Para el consumo promedio se obtuvo una media de 1.75 con una desviación de 0.69 y una media de 1.97 con una desviación típica de 0.78 para la rotación. A partir de estos datos, se logra establecer los intervalos de corte, distribuidos en tres puntos, los cuales fueron categorizados en alto, medio o bajo. La tabla 5 muestra los datos obtenidos para cada variable. Con estos datos se puede determinar cuál es la percepción de la gente sobre los criterios que se proponen para las ventas, cuando esta se mide a partir de juicios que influyen en la decisión de compra de un producto y un servicio.

Posteriormente, con estos resultados se logra identificar la relación que existe entre las dos variables, donde, por medio de la prueba de Chi Cuadrado de Pearson se contrarresta la hipótesis planteada. Como resultado se obtuvo una relación significativa de 107.409, con un valor de $p=.000$. El valor de $p$ es menor a alfa, siendo $a=0.05$. De ahí, es posible concluir que existe una relación entre el consumo promedio y la rotación durante el proceso de ventas.

Para corroborar la hipótesis alcanzada según los datos de Chi Cuadrado de Pearson se procede a identificar del mismo modo la correlación según el coeficiente $V$ de Cramer; como resultado se determina, de igual forma, que sí existe una relación significativa. Tomando en consideración que la V de Cramer, para que sea una relación significativamente intensa debe ser igual o mayor a 0.6. Para esta investigación alcanza un valor de 0.381 , que es considerado en ciencias sociales como una correlación significativa baja, con un nivel de significancia de .000 siendo $p>a$. Por lo tanto, se aprueba la hipótesis. La tabla 6 muestra la relación significativa entre estos dos enfoques.

Partiendo de los valores obtenidos, se logra identificar el promedio y porcentaje que se alcanzó en el estudio de mercado según la valoración realizada a cada criterio, según las distintas escalas formuladas para las preguntas tipo Likert de cinco posiciones y de igual forma el alcance de cada variable. Con estos datos se pueden identificar cuáles poseen mayor rele-

\section{TABLA 5}

VALORES DE LA MEDIA Y DESVIACIÓN ESTÁNDAR

\begin{tabular}{|c|c|c|c|c|c|c|}
\hline & & Percepción & $\begin{array}{l}\text { Consumo } \\
\text { promedio }\end{array}$ & Relación & Rotación & Relación \\
\hline \multicolumn{2}{|l|}{ Media } & & 1.75 & & 1.97 & \\
\hline \multicolumn{2}{|c|}{ Desviación típica } & & 0.69 & & 0.78 & \\
\hline \multicolumn{2}{|l|}{ Mínimo } & & 1 & & 1 & \\
\hline \multicolumn{2}{|l|}{ Máximo } & & 3 & & 3 & \\
\hline \multirow[t]{3}{*}{ Válidos } & Primero & bajo & & 144 & & 106 \\
\hline & Segundo & medio & & 170 & & 145 \\
\hline & Tercero & alto & & 55 & & 118 \\
\hline
\end{tabular}

Fuente: Elaboración propia 
TABLA 6 RELACIÓN ENTRE EL CONSUMO PROMEDIO Y LA ROTACIÓN

\begin{tabular}{|l|l|l|l|} 
& $\begin{array}{l}\text { Unidad de } \\
\text { análisis }\end{array}$ & Relación & Significancia* \\
\hline $\begin{array}{l}\text { Chi Cuadrado } \\
\text { de Pearson }\end{array}$ & 369 & $107.409($ d.f. $=4)$ & 0.000 \\
\hline V de Cramer** & 369 & 0.381 & 0.000 \\
\hline
\end{tabular}

Nota: ${ }^{A}$ Análisis con diferencia significativa de $p<.05 * *$ Aceptado si V de Cramer es $>$ a 0.3 0 de preferencia $>$ a 0.6 siendo el valor máximo 1.

Fuente: Elaboración propia.

vancia en las ventas; estos valores pueden servir como directriz para tomar decisiones a futuro por parte de la empresa. La tabla 7 muestra los resultados obtenidos.

Por otro lado, se procedió también a identificar la opinión que tienen los encuestados sobre cada uno de los criterios considerados para evaluar la relación entre la rotación y el consumo promedio. Por medio de un análisis estadístico descriptivo, aplicando el programa SPSS se elaboraron diversas tablas de contingencia obteniendo los siguientes resultados.

En la tabla 8 se puede observar los resultados obtenidos de la relación entre el sexo y la rotación. De la base de 370 personas (149 mujeres y 221 hombres), se consideraron aspectos como: tiempo, diseño, atención al cliente, ubicación del establecimiento, infraestructura física del local, calidad en el producto y servicio, la cantidad de comida o tamaño de porción que lleva un plato, el precio, las promociones y la constante innovación. El criterio que tuvo mayor valor fue el diseño con un 74\% y un $72 \%$ para el sexo femenino y masculino respectivamente.

A este criterio le sigue la atención y el tiempo de espera, con valores muy similares comprendidos entre el $66 \%$ al $69 \%$ y la calidad del producto con un $62 \%$ para el sexo femenino y un $63 \%$ por parte del masculino seguido de la innovación y cantidad. Si bien la calidad en el producto es considerado en los textos como uno de los
TABLA 7

\section{ANÁLISIS DE PROMEDIOS SEGÚN EL CRITERIO DE VENTAS}

\begin{tabular}{|c|c|c|}
\hline Ítem & Promedio* & Porcentaje \\
\hline Ventas & 1.5 & $90 \%$ \\
\hline Consumo Promedio & 1.6 & $88 \%$ \\
\hline Infraestructura versus precio & 1.49 & $82 \%$ \\
\hline Calidad del producto versus precio & 1.62 & $88 \%$ \\
\hline Oferta gastronómica versus precio & 1.71 & $86 \%$ \\
\hline Rotación & 1.5 & $90 \%$ \\
\hline Diseño & 1.29 & $94 \%$ \\
\hline Tiempo de espera & 1.40 & $92 \%$ \\
\hline Atención en el servicio & 1.37 & $93 \%$ \\
\hline Ubicación geográfica & 1.68 & $86 \%$ \\
\hline Infraestructura interna y externa & 1.63 & $87 \%$ \\
\hline Calidad del producto & 1.46 & $91 \%$ \\
\hline Cantidad de producto a ofrecer & 1.46 & $91 \%$ \\
\hline Precio de un producto y servicio & 1.52 & $90 \%$ \\
\hline Promociones & 1.68 & $86 \%$ \\
\hline Constantes innovaciones & 1.52 & $90 \%$ \\
\hline
\end{tabular}

Nota: *Valores obtenidos donde 5 es considerado totalmente de acuerdo y 1 totalmente desacuerdo.

Fuente: Elaboración propia.

factores más importantes a la hora de elegir un producto o servicio, el mercado quiteño no lo considera como su primera opción al momento de elegir un establecimiento. Como factores de menos importancia se encuentran la ubicación y la infraestructura. Un dato muy importante es que, un 50\% de los hombres consideran que las promociones no influyen sobre el precio en un establecimiento de alimentos y bebidas, por lo tanto este no es un criterio que pueda influir en el consumo promedio. 
TABLA 8

\section{CRITERIOS CONSIDERADOS INFLUYENTES EN LA ROTA- CIÓN SEGÚN EL SEXO DE LA PERSONA}

\begin{tabular}{|c|c|c|c|}
\hline Variables & \multicolumn{2}{c}{ Sexo } & Total \\
\hline & Femenino & Masculino \\
\hline Tiempo & $66 \%$ & $68 \%$ & $67 \%$ \\
\hline Diseño & $74 \%$ & $72 \%$ & $73 \%$ \\
\hline Atención & $69 \%$ & $69 \%$ & $69 \%$ \\
\hline Ubicación & $52 \%$ & $51 \%$ & $51 \%$ \\
\hline Infraestructura & $51 \%$ & $57 \%$ & $55 \%$ \\
\hline Calidad & $62 \%$ & $63 \%$ & $63 \%$ \\
\hline Cantidad & $61 \%$ & $61 \%$ & $61 \%$ \\
\hline Precio & $57 \%$ & $57 \%$ & $57 \%$ \\
\hline Promoción & $56 \%$ & $50 \%$ & $52 \%$ \\
\hline Innovación & $65 \%$ & $61 \%$ & $63 \%$ \\
\hline MUESTRA TOTAL & 149 & 221 & 370 \\
\hline
\end{tabular}

Nota: Elaborados con una base de 370 personas: 149 personas de sexo femenino, 22 personas de sexo masculino

Fuente: Elaboración propia.

En la tabla 9, donde se analiza la relación entre la rotación y la edad de las personas, se visualiza que el criterio diseño, atención y tiempo cuentan con mayor representatividad. El rango de edad de entre 35 a 40 años y el de 41 a 45 años consideran el diseño con mayor relevancia, mientras que el rango de 31 a 35 años y el de 26 a 30 mencionan menor importancia sobre el mismo; esto quiere decir que a mayor edad las personas valoran más esta variable.

Por otro lado, los rangos que demuestran más interés en la variable atención son los que se encuentran entre los 35 a 40 años, 21 a 25 años y el de 41 a 45 años; mientras que, los rangos de 26 a 30 y más de 45 años indican que tiene menor importancia con respecto a la variable atención. Es decir en este criterio para la mayor parte de los encuestados, la atención sí es una variable importante para que vuelvan a un establecimiento de alimentos y bebidas.

La variable tiempo es más representativa para los rangos de 41 a 45 años y para los rangos de 21 a 25 años y de hasta 20 años con el 75\% de participación; mientras que, para los rangos entre 35 a 40 años y más de 45 años es considerado con menor importancia. Como se visualiza, las diferencias entre los rangos son mínimas, esto quiere decir que para la mayor parte de los encuestados este criterio es importante al momento de regresar a un establecimiento de alimentos y bebidas.

Finalmente, se pueden apreciar los resultados obtenidos sobre la calidad del producto con un $63 \%$, compitiendo el cuarto puesto con la innovación, los cuales obtienen valores similares según su sexo. De estos valores, la variable de calidad es la más relevante, sobre todo por los resultados obtenidos del mercado comprendido entre 26 y 30 años, los cuales no consideran importante este factor a la hora de elegir un establecimiento y que no influye en la rotación de la empresa; muy a diferencia de quienes poseen más de 41 años, donde un 71\% lo considera como un factor clave para la rotación.

En la tabla 10 se visualiza la relación entre la rotación en los servicios de alimentos y bebidas y el nivel de estudio de las personas encuestadas. Para las personas que cuentan con estudios técnicos y de secundaria, el diseño es muy importante, mientras que las personas que cuentan con estudios de primaria y universitaria consideran a este criterio con menor relevancia.

Con respecto a la variable atención, las personas que cuentan con estudios técnicos y universitarios mencionan esta variable con mayor relevancia, mientras que las personas con estudios de primaria y secundaria indican tener menor importancia por este criterio. Esto quiere decir que las personas que cuentan con estudios más altos valoran más la atención de los prestadores 
TABLA 9

CRITERIOS CONSIDERADOS INFLUYENTES EN LA ROTACIÓN SEGÚN LA EDAD DE LA PERSONA

\begin{tabular}{|c|c|c|c|c|c|c|c|c|c|}
\hline Variables & \multicolumn{1}{|c|}{ Hasta 20 } & $\mathbf{2 1 - 2 5}$ & $\mathbf{2 6 - 3 0}$ & $\mathbf{3 1 - 3 5}$ & $\mathbf{3 5 - 4 0}$ & $\mathbf{4 1 - 4 5}$ & Más de 45 & Total \\
\hline Tiempo & $73 \%$ & $73 \%$ & $59 \%$ & $59 \%$ & $62 \%$ & $75 \%$ & $64 \%$ & $67 \%$ \\
\hline Diseño & $69 \%$ & $78 \%$ & $68 \%$ & $63 \%$ & $85 \%$ & $79 \%$ & $73 \%$ & $73 \%$ \\
\hline Atención & $69 \%$ & $75 \%$ & $61 \%$ & $63 \%$ & $77 \%$ & $75 \%$ & $59 \%$ & $69 \%$ \\
\hline Ubicación & $47 \%$ & $59 \%$ & $42 \%$ & $51 \%$ & $50 \%$ & $63 \%$ & $55 \%$ & $51 \%$ \\
\hline Infraestructura & $52 \%$ & $65 \%$ & $34 \%$ & $54 \%$ & $69 \%$ & $71 \%$ & $59 \%$ & $55 \%$ \\
\hline Calidad & $65 \%$ & $67 \%$ & $51 \%$ & $68 \%$ & $58 \%$ & $71 \%$ & $64 \%$ & $63 \%$ \\
\hline Cantidad & $59 \%$ & $61 \%$ & $45 \%$ & $68 \%$ & $69 \%$ & $75 \%$ & $73 \%$ & $60 \%$ \\
\hline Precio & $56 \%$ & $62 \%$ & $42 \%$ & $61 \%$ & $65 \%$ & $67 \%$ & $64 \%$ & $57 \%$ \\
\hline Promoción & $49 \%$ & $57 \%$ & $49 \%$ & $44 \%$ & $62 \%$ & $67 \%$ & $45 \%$ & $52 \%$ \\
\hline Innovación & $63 \%$ & $72 \%$ & $53 \%$ & $54 \%$ & $62 \%$ & $63 \%$ & $73 \%$ & $63 \%$ \\
\hline MUESTRA TOTAL & 81 & 102 & 74 & 41 & 26 & 24 & 22 & 370 \\
\hline
\end{tabular}

Nota: Elaborados con una base de 370 personas: 81 personas con una edad de hasta 20 años, 102 personas entre 21 y 25 años, 74 personas entre 26 a 30 años, 41 personas entre 31 a 35 años, 26 personas de 35 a 40 años, 24 personas entre 41 a 45 años y 22 personas con más de 45

Fuente: Elaboración propia.

TABLA 10

CRITERIOS CONSIDERADOS INFLUYENTES EN LA ROTACIÓN SEGÚN EL NIVEL DE ESTUDIOS EN LA PERSONA

\begin{tabular}{|c|c|c|c|c|c|}
\hline \multirow{2}{*}{ Variables } & \multicolumn{5}{|c|}{ Nivel de estudio } \\
\hline Tiempo & Primaria & Secundaria & Técnica & Universitaria & Total \\
\hline Diseño & $33 \%$ & $56 \%$ & $72 \%$ & $69 \%$ & $67 \%$ \\
\hline Atención & $58 \%$ & $60 \%$ & $79 \%$ & $58 \%$ & $65 \%$ \\
\hline Ubicación & $42 \%$ & $60 \%$ & $68 \%$ & $73 \%$ & $69 \%$ \\
\hline Infraestructura & $42 \%$ & $51 \%$ & $52 \%$ & $58 \%$ & $55 \%$ \\
\hline Calidad & $75 \%$ & $44 \%$ & $62 \%$ & $67 \%$ & $63 \%$ \\
\hline Cantidad & $50 \%$ & $44 \%$ & $59 \%$ & $66 \%$ & $61 \%$ \\
\hline Precio & $67 \%$ & $51 \%$ & $58 \%$ & $57 \%$ & $57 \%$ \\
\hline Promoción & $50 \%$ & $49 \%$ & $56 \%$ & $51 \%$ & $52 \%$ \\
\hline Innovación & $42 \%$ & $62 \%$ & $63 \%$ & $64 \%$ & $63 \%$ \\
\hline MUESTRA TOTAL & 12 & 45 & 111 & 202 & 370 \\
\hline
\end{tabular}

Nota: Elaborados con una base de 370 personas: 12 personas con educación primaria, 45 personas con nivel secundario, 111 personas con nivel técnico, y 202 con una educación de nivel universitario.

Fuente: Elaboración propia 
de servicio. Al analizar el tiempo como variable, se puede observar que las personas con estudios técnicos y universitarios valoran el tiempo de servicio mientras que para las personas con estudios de primaria y secundaria no prima este criterio.

Finalmente, como otro resultado obtenido según el criterio de la rotación, se pueden apreciar, en la tabla 11, datos obtenidos durante el estudio, donde del total de personas encuestadas, el 73\% afirma que el diseño del establecimiento es el aspecto más importante para asistir a un restaurante; el 69\% señala que la atención es el segundo criterio; en tercer lugar, con un 67\%, establecen que el tiempo de servicio; en cuarto lugar, con un $63 \%$, se reconoce la calidad como un elemento importante; en quinto lugar, con el 63\%, está la innovación. Las promociones que ofrece el establecimiento es el penúltimo criterio, con el 52\% de aceptación y, finalmente, la ubicación con un 51\% es el criterio más bajo que un consumidor considera para visitar un establecimiento.
Dentro de la misma tabla 11 se puede apreciar la aceptación según la ocupación de la persona; por ejemplo, en la variable diseño, un $84 \%$ de los empleados públicos consideran a este criterio como el más importante al momento de tomar la decisión de asistir a un determinado restaurante. El 69\% de los empleados privados consideran a este criterio como el menos importante para visitar un establecimiento.

El segundo criterio más valorado es la atención; son los empleados públicos en un $77 \%$ quienes definen a la atención como un factor determinante al momento de visitar un restaurante. Por su lado, las personas que no trabajan, representadas por el $66 \%$ del total del segmento analizado, no lo consideran como tal. El tercer lugar lo ocupa la variable del tiempo de servicio como el criterio determinante para visitar un establecimiento; dentro del cual, los empresarios con un $75 \%$ son quienes valoran en mayor grado este criterio para elegir un establecimiento; mientras

\section{TABLA 11}

CRITERIOS CONSIDERADOS INFLUYENTES EN LA ROTACIÓN SEGÚN LA OCUPACIÓN DE LA PERSONA

\begin{tabular}{|c|c|c|c|c|c|}
\hline \multirow{2}{*}{ Variables } & \multicolumn{5}{c}{ Ocupación } \\
& $\begin{array}{c}\text { Empre- } \\
\text { sario }\end{array}$ & Público & Privado & No trabaja & Total \\
\hline Tiempo & $75 \%$ & $71 \%$ & $62 \%$ & $69 \%$ & $67 \%$ \\
\hline Diseño & $79 \%$ & $85 \%$ & $69 \%$ & $70 \%$ & $73 \%$ \\
\hline Atención & $67 \%$ & $77 \%$ & $69 \%$ & $66 \%$ & $69 \%$ \\
\hline Ubicación & $54 \%$ & $55 \%$ & $53 \%$ & $48 \%$ & $51 \%$ \\
\hline Infraestructura & $54 \%$ & $65 \%$ & $53 \%$ & $52 \%$ & $55 \%$ \\
\hline Calidad & $58 \%$ & $71 \%$ & $60 \%$ & $63 \%$ & $63 \%$ \\
\hline Cantidad & $71 \%$ & $72 \%$ & $50 \%$ & $63 \%$ & $61 \%$ \\
\hline Precio & $58 \%$ & $66 \%$ & $54 \%$ & $55 \%$ & $57 \%$ \\
\hline Promoción & $33 \%$ & $54 \%$ & $58 \%$ & $51 \%$ & $52 \%$ \\
\hline Innovación & $63 \%$ & $68 \%$ & $58 \%$ & $64 \%$ & $63 \%$ \\
\hline MUESTRA TOTAL & 24 & 65 & 118 & 163 & 370 \\
\hline
\end{tabular}

Nota:Elaborados con una base de 370 personas: 24 empresarios, 65 personas que trabajan en el sector público, 118 que trabajan en el sector privado, y 163 que no trabajan Fuente: Elaboración propia. 
que los empleados privados, con un $62 \%$ son los que menos lo valoran.

Analizando la variable ocupación; es decir, a qué se dedica el universo encuestado, los empresarios consideran el diseño del restaurante como el criterio más influyente al momento de tomar la decisión para visitarlo, mientras que las promociones son el criterio menos valorado para hacerlo. Del total de empleados públicos encuestados, el 85\% considera al diseño del restaurante como un factor importante; mientras que, el 53\% considera a las promociones como el menos importante. El 69\% de los empleados privados opina que son el diseño y la atención los criterios más valorados, mientras que el 50\% considera que la cantidad de producto es un factor muy valorado. Finalmente, del segmento de personas encuestadas que no trabajan, un 70\% considera que el diseño del restaurante es un criterio determinante para visitarlo, mientras que el criterio más bajo de ese segmento es la ubicación, valorado por un 48\% del total encuestado.

En la tabla 12 se puede identificar que, del 100\% de personas encuestadas, el 40\% corresponde al sexo femenino y el $60 \%$ al masculino, donde un $64 \%$ del total de encuestados consideran que la infraestructura del establecimiento es el elemento de mayor motivación al consumo por persona en el restaurante. En segundo lugar, un 59\% pien- sa que es la calidad del producto lo que incide en el consumo en el establecimiento, finalizando con un 49\% que opina que es la oferta del establecimiento quien determina el nivel de consumo de los clientes.

Dentro del criterio infraestructura, como motivador de compra en un restaurante, son los hombres con un 64\% quienes comparten esta opinión, mientras que las mujeres lo hacen en un $63 \%$. Por otro lado, el 66\% de las mujeres considera que la calidad del producto es el elemento más importante al momento de decidir visitar un restaurante, mientras que el $54 \%$ de los hombres encuestados comparten esta opinión. Finalmente el $50 \%$ de las mujeres considera que la diversidad en la oferta es un determinante para acudir a un restaurante, mientras que el $48 \%$ de los hombres piensan de igual forma.

En la tabla 13 se puede visualizar la relación existente entre el consumo promedio de las personas encuestadas y la relación con las edades. Aquí, las personas hasta los 20 años y el rango de 21 a 25 años consideran que sí existe una relación entre la infraestructura y el precio que pagan por un producto o un servicio; mientras que el rango de 26 a 30 años y el de 31 a 35 consideran que esta relación no tiene relevancia para ellos. Con respecto a la relación existente entre la calidad del producto servicio que le brinda un establecimiento y el precio cancelado por este,

TABLA 12

CRITERIOS CONSIDERADOS INFLUYENTES EN EL CONSUMO PROMEDIO SEGÚN EL SEXO DE LA

PERSONA

\begin{tabular}{|c|c|c|c|c|c|}
\hline VARIABLE & CRITERIO & $\begin{array}{l}\text { INFRAESTRUCTURA } \\
\text { INFLUYE EN PRECIO }\end{array}$ & $\begin{array}{l}\text { CALIDAD INFLUYE } \\
\text { EN PRECIO }\end{array}$ & $\begin{array}{l}\text { OFERTA INFLUYE } \\
\text { EN PRECIO }\end{array}$ & $\begin{array}{l}\text { MUESTRA } \\
\text { TOTAL }\end{array}$ \\
\hline \multirow{3}{*}{ sexo } & Femenino & $63 \%$ & $66 \%$ & $50 \%$ & 149 \\
\hline & Masculino & $64 \%$ & $54 \%$ & $48 \%$ & 221 \\
\hline & Total & $64 \%$ & $59 \%$ & $49 \%$ & 370 \\
\hline
\end{tabular}

Nota:Elaborados con una base de 370 personas: 149 personas de sexo femenino, 221 personas de sexo masculino

Fuente: Elaboración propia. 
TABLA 13

CRITERIOS CONSIDERADOS INFLUYENTES EN EL CONSUMO PROMEDIO SEGÚN LA EDAD DE LA

PERSONA

\begin{tabular}{|c|c|c|c|c|c|}
\hline VARIABLE & CRITERIO & $\begin{array}{l}\text { INFRAESTRUCTURA } \\
\text { INFLUYE EN PRECIO }\end{array}$ & $\begin{array}{l}\text { CALIDAD INFLUYE } \\
\text { EN PRECIO }\end{array}$ & $\begin{array}{l}\text { OFERTA INFLUYE } \\
\text { EN PRECIO }\end{array}$ & $\begin{array}{l}\text { MUESTRA } \\
\text { TOTAL }\end{array}$ \\
\hline \multirow{8}{*}{ Edad } & HASTA 20 & $70 \%$ & $68 \%$ & $51 \%$ & 81 \\
\hline & $21-25$ & $69 \%$ & $61 \%$ & $52 \%$ & 102 \\
\hline & $26-30$ & $59 \%$ & $55 \%$ & $50 \%$ & 74 \\
\hline & $31-35$ & $59 \%$ & $56 \%$ & $41 \%$ & 41 \\
\hline & $35-40$ & $65 \%$ & $42 \%$ & $38 \%$ & 26 \\
\hline & $41-45$ & $42 \%$ & $46 \%$ & $38 \%$ & 24 \\
\hline & MÁS DE 45 & $64 \%$ & $64 \%$ & $64 \%$ & 22 \\
\hline & Total & $64 \%$ & $59 \%$ & $49 \%$ & 370 \\
\hline
\end{tabular}

Nota: Elaborados con una base de 370 personas: 81 personas con una edad de hasta 20 años, 102 personas entre 21 y 25 años, 74 personas entre 26 a 30 años, 41 personas entre 31 a 35 años, 26 personas de 35 a 40 años, 24 personas entre 41 a 45 años y 22 personas con más de 45.

Fuente: Elaboración propia.

las personas de hasta 20 años y el rango de más de 45 años consideran con mayor importancia, mientras que los rangos de 35 a 40 años y el rango de 41 a 45 años lo ubican con menor importancia esta relación. Finalmente, el rango de más de 45 años y el rango de 21 a 25 años consideran que, al existir una oferta gastronómica adecuada, las personas se motivan a consumir más y a pagar un mayor precio, mientras que el rango de entre 35 a 40 años y de 41 a 45 años consideran esto pero en menor porcentaje.

En la tabla 14 se pueden apreciar los resultados obtenidos según el nivel de estudio, en donde del total de personas encuestadas, el 3\% corresponde a un mercado con un nivel de estudios primarios, el 12\% secundarios, el 30\% tiene estudios técnicos y 55\% estudios universitarios. De estos, el $75 \%$ de las personas que tienen un nivel de estudios primarios le da mayor valor a la infraestructura del establecimiento frente al precio del producto que van a recibir; el 58\% del segmento valora la calidad del producto con relación al precio mientras que el 50\% le da mayor valor a la variedad de la oferta frente al precio del producto.
Las personas con un nivel de educación técnica y secundaria, valoran, en mayor grado, a la infraestructura del restaurante en relación con el precio de venta y lo hacen en menor grado a la diversidad de la oferta del producto. Las personas con un nivel de educación superior valoran en mayor grado a la infraestructura y a la calidad frente al precio que pagan por un producto, mientras que la oferta lo hacen en menor grado.

En la tabla 15 se pueden apreciar los resultados obtenidos según la ocupación del objeto de estudio. Del total de personas encuestadas, el 6\% son empresarios, el 18\% son empleados públicos, el 31\% son empleados privados, mientras que el $44 \%$ no trabaja.

En relación con el precio de venta de los productos en un restaurante, del total de empresarios encuestados, el 71\% valoran la infraestructura, mientras que en igual porcentaje con un 54\% valoran la calidad de los productos y la variedad de la oferta gastronómica. El 58\% de los empleados públicos valoran más la infraestructura en relación con el precio de venta a diferencia 


\section{TABLA 14 \\ CRITERIOS CONSIDERADOS INFLUYENTES EN EL CONSUMO PROMEDIO SEGÚN EL NIVEL DE ESTUDIOS EN LA PERSONA}

\begin{tabular}{|c|c|c|c|c|c|}
\hline VARIABLE & CRITERIO & $\begin{array}{l}\text { INFRAESTRUCTURA } \\
\text { INFLUYE EN PRECIO }\end{array}$ & $\begin{array}{l}\text { CALIDAD INFLUYE } \\
\text { EN PRECIO }\end{array}$ & $\begin{array}{l}\text { OFERTA INFLUYE } \\
\text { EN PRECIO }\end{array}$ & $\begin{array}{l}\text { MUESTRA } \\
\text { TOTAL }\end{array}$ \\
\hline \multirow{5}{*}{$\begin{array}{l}\text { Nivel de } \\
\text { estudios }\end{array}$} & Primaria & $75 \%$ & $58 \%$ & $50 \%$ & 12 \\
\hline & Secundaria & $51 \%$ & $49 \%$ & $31 \%$ & 45 \\
\hline & Técnica & $66 \%$ & $51 \%$ & $50 \%$ & 111 \\
\hline & Universitaria & $65 \%$ & $65 \%$ & $52 \%$ & 202 \\
\hline & Total & $64 \%$ & $59 \%$ & $49 \%$ & 370 \\
\hline
\end{tabular}

Nota: Elaborados con una base de 370 personas: 12 personas con educación primaria, 45 personas con nivel secundario, 111 personas con nivel técnico, y 202 con una educación de nivel universitario.

Fuente: Elaboración propia.

TABLA 15

CRITERIOS CONSIDERADOS INFLUYENTES EN EL CONSUMO PROMEDIO SEGÚN LA OCUPACIÓN DE LA PERSONA

\begin{tabular}{|c|c|c|c|c|c|} 
VARIABLE & CRITERIO & $\begin{array}{c}\text { INFRAESTRUCTURA } \\
\text { INFLUYE EN PRECIO }\end{array}$ & $\begin{array}{c}\text { CALIDAD INFLUYE } \\
\text { EN PRECIO }\end{array}$ & $\begin{array}{c}\text { OFERTA } \\
\text { INFL PRECIO }\end{array}$ & $\begin{array}{c}\text { MUESTRA } \\
\text { TOTAL }\end{array}$ \\
\hline \multirow{5}{*}{ Ocupación } & Empresario & $71 \%$ & $54 \%$ & $54 \%$ & 24 \\
\cline { 2 - 6 } & Público & $58 \%$ & $49 \%$ & $38 \%$ & 65 \\
\cline { 2 - 6 } & Privado & $61 \%$ & $62 \%$ & $57 \%$ & 118 \\
\cline { 2 - 6 } & No trabaja & $67 \%$ & $61 \%$ & $47 \%$ & 163 \\
\cline { 2 - 6 } & Total & $64 \%$ & $59 \%$ & $49 \%$ & 370 \\
\hline
\end{tabular}

Nota: Elaborados con una base de 370 personas: 24 empresarios, 65 personas que trabajan en el sector público, 118 que trabajan en el sector privado, y 163 que no trabajan.

Fuente: Elaboración propia.

de la variedad de la oferta con una menor valoración. El 62\% de los empleados privados valoran la calidad del producto del restaurante al momento de relacionarlo con el precio de venta, mientras que lo que menos valoran es la variedad de la oferta. Finalmente, el $67 \%$ de las personas que no trabajan valoran en primer lugar a la infraestructura, en segundo lugar a la calidad y, finalmente, a la variedad de la oferta al momento de pagar el precio de un producto del establecimiento.

\section{CONCLUSIONES}

En relación con lo mencionado, se pueden establecer que la presente investigación aporta a los objetivos planteados y sobre todo al cumplimiento de la hipótesis presentada, lo que da un resultado afirmativo sobre la relación que existe entre los factores que influyen en el consumo promedio y los factores que influyen en la rotación para la decisión de compra en restaurantes de la ciudad de Quito ubicados en los sectores del Centro Histórico, República del Salvador, La Mariscal e Iñaquito.

En primer lugar, se tiene los ingresos como la base fundamental del negocio. La persona encargada debe establecer mecanismos y estrategias que permitan al establecimiento contar con los ingresos necesarios para cubrir sus costos y asegurar una rentabilidad razonable que garantice el 
crecimiento del negocio y su permanencia a través del tiempo. Estos ingresos están directamente relacionados con diferentes aspectos del negocio como son el producto, tipo de servicio, ubicación, infraestructura y, sobre todo, con el precio que el cliente está dispuesto a pagar y que debe estar al nivel de los servicios que brinda como parte integral del concepto del negocio.

Asimismo, estos ingresos dependen de forma directa de la variable rotación, de igual forma, garantizan el nivel de ingresos del establecimiento y deben ser de conocimiento de quien gestiona el establecimiento para que cuente con información que le permita establecer estrategias que apunten a subir la rotación como uno de los elementos clave de la generación de ingresos del negocio. La rotación, además, tiene una relación directa con el concepto del negocio y, en alto grado, con la fidelidad de los clientes, quienes valoran el producto y el servicio como elementos de mayor impacto para visitar frecuentemente un establecimiento. Pero, ¿qué hace que un cliente regrese a un restaurante? Entre los criterios que más respuesta se obtuvieron se hallan el diseño del establecimiento, el tiempo de servicio, la atención, la calidad e infraestructura del local.

El segundo elemento que influye de forma directa en la generación de ingresos, en un restaurante, es el consumo promedio, el cual depende de los vendedores (meseros) que ofrecen los productos; lo que lleva a deducir que, si los establecimientos desean incrementar su consumo promedio deben contar con un personal preparado que conozca lo que vende, que tenga la actitud de enfrentar los momentos de conflicto con los clientes y que cuente con una alta capacidad de comunicación que le permita ofrecer productos adicionales a los solicitados por el cliente $y$, al mismo tiempo, que generen una sinergia con el restaurante que permita repetir la compra y generar una lealtad.
Con base en los resultados se podría concluir que uno de los efectos relevantes para que se genere una mayor rotación en un establecimiento de alimentos y bebidas, tiene relación con el diseño, la atención y el tiempo. En el primer caso, mientras más edad tiene la persona, mayor valoración tienen sobre el diseño y este tiene una estrecha relación con las percepciones sensoriales. Por lo tanto, hay que dar mayor énfasis en este factor; por otro lado, las variables atención y tiempo, para casi todos los grupos de personas encuestados, son de relevancia sin importar la edad.

De igual forma, la formación de las persona, mientras más alta es su formación profesional o cuentan con más estudios, tienen una percepción más alta de lo que se refiere a un servicio de calidad en un establecimiento de alimentos y bebidas y esto sucede de la misma manera con respecto al tiempo de atención. Finalmente, con respecto a la ocupación de las personas, se puede ver claramente que los empleados públicos valoran más los criterios como diseño y atención, mientras que para los empresarios el tiempo de servicio prima frente a los empleados públicos, privados y a las personas que no trabajan.

Con respecto a la relación entre el sexo y el consumo promedio de una persona en un establecimiento de alimentos y bebidas, en el caso de las mujeres, ellas opinan mayormente que la calidad del producto y la oferta gastronómica influye en el precio que el establecimiento establezca en su carta, mientras que para los hombres prima la infraestructura del lugar. De acuerdo con la relación entre la edad y el consumo promedio, sin importar la edad, los encuestados consideran una relación entre la infraestructura, calidad y oferta como variables que influyen en el precio en este orden. Si bien la calidad es considerada como un criterio muy importante para que se generen ventas, los resultados de esta investigación muestran todo lo contrario. 
Cuando se analiza el criterio de consumo promedio con la formación de los encuestados se puede ver claramente que en todos los casos indican que la infraestructura si tiene relación con el precio de la oferta gastronómica. En el caso del análisis entre el consumo promedio y la ocupación de los encuestados mencionan que existe una relación entre la infraestructura y el precio que se establezca en una carta; estos tres criterios priman en los empresarios, empleados públicos y las personas que no trabajan.

Se podría concluir que el diseño de un establecimiento influye considerablemente al momento de establecer los precios de la carta mucho más que la calidad u oferta gastronómica. Por lo tanto, se abre campo a futuras líneas de investigaciones más profundas o específicas, en relación con el diseño y las percepciones sensoriales.

\section{REFERENCIAS}

Barrantes, H. (2003). Cálculo integral de una variable. San José, Costa Rica: EUNED.

Baskerville, R., \& Dulipovici, A. (2006). The Theoretical Fundamentals of Library Marketing. Knowledge Management Research \& Practice, 4(2), 83- 105. doi: 10.1057/palgrave.kmrp.8500090

Boullón, R. (2003). Planificación del espacio turístico (3ra. Ed.). Editorial Trillas: México.

Cabrera, S. (2013). La fidelización del cliente en negocios de restauración. La fidelización del cliente en negocios de restauración. Centro de estudios en Diseño y Comunicación, (45), 155 - 164.

Campos, M. D. (2010). Compendio para el estudio de las finanzas corporativas básicas en las Universidades. México: Universidad Iberoamericana.

Cámara de Turismo de Pichincha (CAPTUR), (2002). Alimentos y Bebidas. Recuperado de http://www. captur.com/index.php

Choo, C. W. (2002). Information Management for the Intelligent Organization: The Art of Scanning the Environment. New Jersey: Information Today, Inc..
Cooper, B., Floody, B., \& Mc Neill, G. (2002). Cómo iniciar y administrar un restaurante. Bogotá, Colombia: Grupo Editorial Norma.

Cuevas, F. (2002). Control de Costos en los restaurantes. México: Limusa.

Davis, B., et al. (2012). Food and Beverage Management (5th edition.). New York: Routledge.

De la Parra, E. (2004). El poder de vender. México: Panorama Editorial. S. A.

Dixon, M., Kimes, S. E., \& Verma, R. (2009). Customer Preferences for Restaurant Technology. Cornell University School of Hotel Administration.

Escudero, M. J. (2013). Gestión logística y commercial. Ediciones Paraninfo.

Feijoó, J. L., et al. (2009). Alimentos y Bebidas. Su gerenciamiento en hoteles y restaurantes. Buenos Aires, Argentina: Lectorum- Urgeman.

Fernández, J., et al. (2004). Manual de introducción al análisis contable de la empresa. Madrid: Editorial Complutense.

Fernández, R. (2010). La mejora de la productividad en la pequeña y mediana empresa. Alicante, España: Editorial Club Universitario.

Ferrer, M. A., \& Gamboa C., T. (2005). Indicadores para el control de gestión de procesos básicos en hoteles. Venezuela: Red Actualidad Contable Faces.

Flores, J. (2004). Medición de la efectividad de la cadena de suministro. México: Panorama editorial.

García Ortiz, F., et al. (2011). Operaciones básicas y servicios en restauración y eventos especiales. Madrid, España: Paraninfo.

Goldratt, E. (2009). La Carrera, en busca de las ventajas competitivas. Buenos Aires, Argentina: Editores Granica S. A.

Gomes, A., De Azevedo Barbosa, M. D., \& Gomes de Souza, A. (2013). El sistema de oferta de restaurantes de alta gastronomía; Una perspectiva sensorial de las experiencias de consumo. Estudios y Perspectivas en Turismo, (22), 336 - 356. 
Gudiño, J. (2009). Glosario Contabley Financiero. Bogotá, Colombia: Clásico Digital.

Kahle, L., \& Kennedy, P. (1989). Using the list of values (LOV) to Understand Consumers. Journal of ConsumerMarketing, 6(3), 5 - 12. doi: 10.1108/eb024742

Kandampully, J., Mok, C., \& Sparks, B. (2009). Quality Management in Hospitality, Tourism and Leisure. New York, USA: The Haworth Hospitality Press.

Kotler, P. (2012). Dirección de Marketing. México: Pearson Educación.

León Valbuena, N. (2013). Fuerza de ventas determinante de la competitividad empresarial. Revista de Ciencias Sociales, 19(2), 79-389.

Martínez, J. (2004). Dirección de alimentos y bebidas en hoteles. México: Limusa.

Mataix Verdú, J. (2013). Nutrición para educadores (2a. ed.). Madrid, España: Ediciones Díaz de Santos.

Milliman, R. E. (1986). The Influence of Background Music on the Behavior of Restaurant Patrons. Journal of Consumer Research, 13(2), 286-289.

Ministerio de Turismo. (2013). Catastro. Recuperado de http://www.ute.edu.ec/index.aspx?idSeccion=33\&idCategoria $=99 \& i d P o r t a l=1$

Ministerio de Turismo. (2014). Consolidado Nacional Catastro 2014. Ministerio de Turismo. Quito.

Ninamango Guevara, M. (2012). Marketing. Perú: Gráfica e impresiones Richi.

Njite, D., Dunn, G., \& Kim, L. H. (2008). Beyond good food: what other attributes influence consumer preference and selection of fine dining restaurants. Journal of Foodservice Business Research, 11(2), 236 - 266.

Ocampo, N. (2012). Evaluación Diferencial de la Memoria. Revista de Investigación Psicológica, 7, 43-68.

Oviedo, H., \& Campo, A. (2005). Aproximación al uso del coeficiente alfa de Cronbach. Revista Colombiana de Psiquiatría, 35(4), 572 - 580.

Pedraza, D. (2005). Disponibilidad de alimentos como factor determinante de la Seguridad Alimentaria y Nutricional y sus representaciones en Brasil. Revista de Nutrição, 18(1), 129-143.

Rodés, A. (2014). Gestión económica y Financiera. Madrid, España: Paraninfo.

Rodríguez Franco, J., \& Pierdant Rodríguez, A. I. (2014). Estadística para administración. México: Larousse - Grupo Editorial Patria.

Schmal, R., \& Olave, T. (2014). Optimización del Proceso de Atención al Cliente en un Restaurante durante Períodos de Alta Demanda. Información Tecnológica, 25(4), 27-34.

Secretaría de Territorio, Hábitat y Vivienda - MDMQ. (2010). Indicadores para la planificación territorial. Recuperado de http://sthv.quito.gob.ec/images/ indicadores/Barrios/demografia_barrio10.html.

Segura, M., \& Varó, P. (2010). Manipulador de comidas preparadas. San Vicente (Alicante): ECU.

Suárez, L. P., et al. (2011). La profesión turística: ejes de explicación en su relación con la formación académica. Revista de la educación superior, 40(160), $108-134$.

Vera, J., \& Trujillo, A. (2009). El Papel de la Calidad del Servicio del Restaurante como Antecedente de la Lealtad del Cliente. Panorama Socioeconómico, 27(38), 16- 30.

World Tourism Organization (UNWTO). (2015). UNWTO Annual Report 2014; Tourism's contribution to trade. World Tourism Organization (UNWTO). Madrid.

Zamora, J., Vásquez, A., Díaz, R., \& Grandón, M. (2011). Hacia la comprensión de la lealtad del cliente de restaurantes: Caso Talca, Región del Maule - Chile. Estudios y Perspectivas en Turismo, 20(3), 563- 583.

Recibido: 6 de setiembre de 2017

Aceptado: 15 de febero de 2018 